\title{
Artificial Intelligence in Pharmaceutical Research \& Development
}

Dr John Mitchell, EaStCHEM School of Chemistry and Biomedical Sciences Research Complex, University of St Andrews, St Andrews KY16 9ST, UK

The year 2018 has so far been a turbulent time for artificial intelligence (Al). A fatal crash involving a driverless car combined with a scandal where personal data from social media were allegedly repurposed for political and financial gain, and a computer algorithm failing to invite 450,000 English women to breast cancer screening, generating a feeding frenzy for cybersceptics [1]. Stories of international hacking, whether by foreign governments, criminal gangs, or bored teenagers, have never been far from the printed or web pages of the news outlets. [2] Despite the hype and scaremongering surrounding their coverage, these recent developments highlight the need for serious thinking about the future of both the internet and Al. Amongst the questions raised is this; what do we, and what should we, use the enormous power of modern computers and networks for?

In the pharmaceutical industry, current practice regarding the use of in silico brainpower can seem mundane. Computational chemistry is sufficiently well established that few doubt its roles in compound discovery and academic research. Find me some library compounds similar to that lead [3]; run a QM/MM calculation of the enzyme active site [4]; predict the aqueous solubilities of this series of compounds [5]. These are all acknowledged as tasks for the computer. In chemistry, machine learning $(\mathrm{ML})$ is widely used to calculate, or more realistically estimate, those molecular properties too complex or too expensive to compute from quantum mechanics; it is old news that QSAR has become ML [6]. Yet within the whole process of discovering new scientific knowledge, it is mostly specific and relatively restricted technical functions that are executed in silico [7].

In a world where Al can diagnose, monitor, and help us understand diseases, [8,9] drive cars [10], trade currencies, place bets, play chess better than Garry Kasparov or Magnus Carlsen and Go better than Lee Sedol, what other activities in medicinal chemistry could be enhanced by Al? Pretty much any area requiring patterns to be identified and connections found in large datasets is ripe for addressing in silico. Creativity, nimbleness, and perhaps desperation to find a successful new niche, have led many newer, smaller and less well-known companies to explore the possibilities. Euretos are using large 'omics datasets to search for links between molecular mechanisms and diseases, and have recently announced a tie-in with scientific publisher Elsevier to add Al-generated hypotheses to research papers $[11,12]$. Sparrho aim to help the scientific community by using both Al and human editors to extract and curate the most significant scientific research findings from the increasingly vast research literature [13]. BioXcel [14] and Qrativ [15] are respectively using Al to repurpose existing drugs and to find treatments for unmet medical needs. The pharma.ai project involves the company Insilico Medicine, who have a particular focus on aging research, and aims to provide deep learning neural networks as solutions to a range of needs across the pharmaceutical industry. Datavant are improving the management and integration of clinical trial data with Al. These are a few examples from the dozens of, mostly new, companies working on Al-related projects with likely applications in drug design and development. They span a wide range of activities within pharmaceutical research, suggesting that the future may involve Al playing multiple different roles in the process of drug development and in the increasing personalisation of medical treatment. 
The future of Al for drug discovery

Now that Al is here, it is hard to see how the genie is going to be put back into the bottle. If $\mathrm{Al}$ is seen to provide a competitive advantage for drug discovery, then it is likely to be rapidly and widely adopted. The consequences of the industry adapting to embrace that new reality will inevitably play themselves out, and late adopters will feel the pressure to embrace the new ways of working for fear of falling out of the race. If any larger and more established players still lag behind, they will need to adapt or perish.

The booming and looming presence of Al promises, or threatens, to transform the ways in which science, business, and indeed society, work. At least up until now, in silico intelligence has mostly been targeted at very specific and technical tasks, whether chess playing, quantum chemistry or bluffing a way through the Turing test [16]. The outstanding performance of computers in very specific activities cannot mask the absence of general-purpose near-human level silicon intelligence. However, even if Al for the time being needs to be substantially redesigned, reprogrammed and retrained for each new job, what role might future Al play in managing projects and making business decisions? In the short term, Al will cover a wider selection of tasks within projects, linking together large quantities of data from disparate sources to discover new knowledge and to suggest unexpected routes forward. Such as, that could be an excellent drug for a quite different indication, or perhaps this disease is closely linked to that one and the separate projects should be merged. This is pretty much what the start-up companies are already doing [11]. Maybe Al can even write up research papers, as sciNote's Manuscript Writer is claimed to do [17]?

The longer term prospects for organisations are intriguing. While scientists and doctors are already having to learn how to improve performance by sharing parts of their traditional functions with Al, how might senior managers and CEOs face up to the same prospect? Maybe a computer can make decisions about which projects to fund or disband, which sites to open or close, which competitors to merge with or fight, and do so better than a human manager? As Taylor has recently pointed out [18], Al also has the ability to dramatically alter pharma's intellectual property (IP) landscape. The capacity of powerful new algorithms to extract knowledge by aggregating disparate sources of data raises the question of how, and under what conditions, companies should make data available electronically. Data around the internet, for instance in open access publications, may prove more valuable to competitors in the Al era than they would have done a decade ago. On the other hand, if Al discovers useful knowledge without significant human intervention, to what extent should the IP of that discovery be legally protectable?

The problem of perception

The wider societal narrative will revolve around how humans can work together with Al, without ceding control. Optimists believe that computers can improve our lives, open up new possibilities, 
discover new medicines and treatments, and relieve the burden of mundane tasks. Pessimists may fear the sci-fi nightmare that we are sleepwalking into a takeover by intelligences that will soon outstrip our own [19]. In a rapidly and fundamentally changing environment, the fear that my job, privacy and freedom are under threat from the robots, data harvesters and algorithms cannot easily be avoided. [2] In mundane ways, many of us are affected by the computer says no scenarios of being turned down for a loan or denied passage through a ticket barrier by a machine that, we may feel, fails to recognise our rights and is harder to argue with than the worst of jobsworths. Nonetheless, humans can be inconsistent and fickle in our attitudes to Al technology. Few of those people who are outraged by alleged violations of data privacy are likely to abandon major social media platforms in protest. Most of us simply click through the online terms and conditions as quickly as possible.

However, the pharma industry has a big advantage that most other actors do not. The promise of medicines to heal our diseases is as strong as ever in a society where surviving into older age may bring even more medical conditions into play. Medical researchers can and should be seen as a force for good. If the public believe that Al discovers medicines, accurately diagnoses diseases, and thus helps prolong active and healthy life, then a more Al-focused pharmaceutical industry should prove widely acceptable. Nonetheless, this goodwill cannot be presumed upon. Public reaction to abuse of, theft or loss of, or undue profiteering from patient data could be very negative. [2] Politicians walk a tightrope of pandering to often fickle public opinion while trying to create a legal and regulatory environment that will foster innovation and growth. Even though the age of Al has already begun, society is miles from resolving the practical, legal and ethical issues involved.

Conclusion

In the short term, we can expect to see even more small biotech and software companies exploring ways of leveraging Al to address particular niches in the drug discovery and development pipeline. While many will either fail or run out of money, others are likely to succeed well enough to become sustainable, and probably to be acquired by bigger players. If Al does indeed help to discover drugs, as I expect that it will, in the medium term it will be increasingly integrated into the working practices of organisations both large and small. It will also change the way we do academic science, with patterns within and connections between data being discovered automatically in large quantities. It is certainly necessary to think about effects on employment; many jobs will change, some undoubtedly disappear, and in all likelihood new kinds of role for researchers and managers interfacing with Al will be created. Recent technological advances, from the Human Genome Project to high throughput screening, have been sold on their ability to discover more, better, and often more personalised medicines. Al will be no exception is this regard, and indeed it offers the prospect of leveraging the other advances of the last 20 years or so to improve the productivity of the industry and better meet patients' needs. Nevertheless, there are justifiable fears about potential data misuse, about the balance between $\mathrm{Al}$ and human control, and about the impact on employment and on employees' roles in the organisation. 
Most musings about of the future of technology turn out to be wrong, with the odd noble exception like David Gerrold's famous and uncannily accurate 1999 prediction of the modern smartphone [20]. While it is a safe bet that resources will continue to be poured into Al development, it is not clear whether the changes will be evolutionary or revolutionary. At least for now, it still seems reasonable to believe that we can expect in future to use $\mathrm{Al}$ as a tool for discovering medicines, amongst many other tasks both worthy and trivial, without being doomed to live in a robot controlled dystopia.

References

1 Levin S (2018) Uber crash shows 'catastrophic failure' of self-driving technology, experts say, The Guardian 22 March 2018 https://www.theguardian.com/technology/2018/mar/22/self-driving-car-uber-death-womanfailure-fatal-crash-arizona

2 TIME Cybersecurity: Hacking, the Dark Web and You, TIME, New York (2018) ISBN 978-1547842407

3 Bender A, Glen RC. Molecular similarity: a key technique in molecular informatics. Organic \& Biomolecular Chemistry, 2(22):3204-3218 (2004) doi: 10.1039/b409813g

4 Ahmadi S, Barrios Herrera L, Chehelamirani M, Hostas J, Jalife S, Salahub DR. Multiscale modeling of enzymes: $\mathrm{QM}$-cluster, $\mathrm{QM} / \mathrm{MM}$, and $\mathrm{QM} / \mathrm{MM} / \mathrm{MD}$ : A tutorial review. International Journal of Quantum Chemistry, 118(9):e25558+ (2018) doi: 10.1002/qua.25558

5 Bergstrom CAS, Larsson P. Computational prediction of drug solubility in water-based systems: Qualitative and quantitative approaches used in the current drug discovery and development setting. International Journal of Pharmaceutics, 540(1-2):185-193 (2018) doi: 10.1016/j.ijpharm.2018.01.044

6 Lavecchia A (2015) Machine-learning approaches in drug discovery: methods and applications. Drug

Discovery Today 20 (3):318-331. 10.1016/j.drudis.2014.10.012

7 Sliwoski G, Kothiwale S, Meiler J, Lowe EW (2013) Computational Methods in Drug Discovery,

Pharmacological Reviews 66 (1):334-395. doi: 10.1124/pr.112.007336

8 Hsu J (2017), Can a Crowdsourced Al Medical Diagnosis App Outperform Your Doctor? Scientific American 11 August 2017, https://www.scientificamerican.com/article/can-a-crowdsourced-ai-medical-diagnosis-appoutperform-your-doctor/

9 Hamet P, Tremblay J (2017) Artificial intelligence in medicine. Metabolism 69:S36-S40 doi:

10.1016/i.metabol.2017.01.011

10 Ryan A (2015) Kant is my co-driver: philosophy and driverless cars, THES 30 July 2015,

https://www.timeshighereducation.com/opinion/philosophy-and-driverless-cars-kant-is-my-my-co-driver

11 Smith S (2018) https://blog.benchsci.com/startups-using-artificial-intelligence-in-drug-discovery

12 Boersma H (2018) Elsevier press release, 6 Feb 2018 https://www.elsevier.com/about/press-

releases/science-and-technology/elsevier-and-euretos-start-innovative-pilot-to-bring-ai-derived-hypotheses-

into-the-scholarly-dialog

13 Magistretti B (2017) https://venturebeat.com/2017/07/10/sparrho-raises-3-million-to-democratize-accessto-scientific-research/

14 https://www.nasdaq.com/article/ai-based-drug-discovery-biotech-bioxcel-therapeutics-sets-terms-for-60million-ipo-cm927198

15 https://medtechboston.medstro.com/blog/2017/09/22/qrativ-combines-ai-and-big-data-to-discover-newpurposes-for-drug-treatments/

16 You J (2015) Beyond the Turing test, Science 347 (6218) 116 doi: 10.1126/science.347.6218.116

17 Pellis R (2017) Rise of the research bots: Al software that writes your papers for you, THES 22 November

2017. https://www.timeshighereducation.com/news/rise-research-bots-ai-software-writes-your-papers-you

18 Taylor C (2018) Al meets IP, Pharma Times

http://www.pharmatimes.com/magazine/2018/may 2018/ai and ip

19 Müller VC (2014) Risks of general artificial intelligence, Journal of Experimental \& Theoretical Artificial

Intelligence 26 (3):297-301 doi: 10.1080/0952813X.2014.895110

20 Gerrold D (1999) Is that a Pita in your pocket? Smart Reseller 20 December 1999 